\title{
Implantable left ventricular assist device for patients with postinfarction ventricular septal defect
}

\author{
Cristiano Faber, MD, Patrick M. McCarthy, MD, Nicholas G. Smedira, MD, James B. Young, MD, Randall C. Starling, MD,
} and Katherine J. Hoercher, RN, Cleveland, Ohio

$\mathrm{T}$ he mortality for patients with postinfarction ventricular septal defect (VSD) remains high because of extensive ventricular infarction and cardiogenic shock. ${ }^{1}$ Attempts to bridge patients with VSD to transplantation are complicated by technical difficulties because of necrotic muscle at the site of left ventricular apical cannulation and previously placed patches for VSD repair. We report 2 patients with cardiogenic shock after VSD repair who were bridged to transplant with implantable left ventricular assist device (LVAD) support.

\section{Clinical Summaries}

PATIENT 1. A 57-year-old man was transferred 2 days after an anterior wall myocardial infarction with a large left-to-right shunt on an intra-aortic balloon pump and inotropic support. The anterior VSD was repaired with a bovine pericardial patch similar to the technique of David et al. ${ }^{2}$ The postoperative course was complicated by ongoing cardiogenic shock, despite maximal inotropic and intra-aortic balloon pump support. Despite no residual shunt, the patient had impending multiple organ failure. Seven days after VSD repair, a HeartMate pneumatic LVAD (Thermo Cardiosystems, Inc, Woburn, Mass) was implanted. At the operation, the left ventricle was opened at the apex, leaving the patch undisturbed on the septum. The inflow cannula cuff was sutured to the apex of the left ventricle with horizontal mattress sutures and buttressed with a strip of felt, with the inflow cannula oriented toward the mitral valve. The patient was weaned off inotropic drugs with right ventricular function preserved. He was transferred to a nursing floor, and the only postoperative complication was a Staphylococcus aureus infection, which was successfully treated with antibiotics. LVAD explantation and cardiac transplantation were performed on the 73rd day of LVAD support, and the patient was discharged 10 days after transplantation. Five years after transplantation, the patient is alive and well.

PATIENT 2. A 54-year-old man underwent emergency anterior VSD patch closure and coronary artery bypass. His postoperative

From the Departments of Cardiology and Thoracic and Cardiovascular Surgery, Kaufman Center for Heart Failure, Cleveland Clinic Foundation, Cleveland, Ohio.

Received for publication Nov 26, 2001; accepted for publication Feb 16, 2002.

Address for inquiries: Patrick M. McCarthy, MD, Department of Thoracic and Cardiovascular Surgery, The Cleveland Clinic Foundation, 9500 Euclid Ave, F25, Cleveland, OH 44195 (E-mail: mccartp@ccf.org).

J Thorac Cardiovasc Surg 2002;124:400-1

Copyright (C) 2002 by The American Association for Thoracic Surgery

$0022-5223 / 2002 \$ 35.00+0 \quad \mathbf{1 2 / 5 4} / \mathbf{1 2 4 2 4 3}$

doi:10.1067/mtc. 2002.124243 convalescence was complicated by congestive heart failure, but the patient eventually was transferred to a rehabilitation facility. He was transferred to our hospital because of persistent congestive heart failure and was listed for transplantation. He had progressive deterioration requiring 2 inotropic drugs, and a Novacor LVAD (World Heart Corp, Ottawa, Ontario, Canada) was implanted. The left ventricular apex was opened, and the patch was left on the septum. The inflow cannula was placed and secured with the same technique used in patient 1 . After a slow recovery caused by cardiac cachexia, he was discharged from the hospital. His postLVAD course was complicated by a transient ischemic attack and high panel-reactive antibody titers. On postoperative day 91 after LVAD implantation, the patient underwent LVAD explantation and cardiac transplantation. The patient was discharged home 13 days after transplantation and is alive and well 3 years after the operation.

\section{Discussion}

In the postthrombolytic era the mortality of postinfarction VSD continues to be high: $94 \%$ at 30 days if treated medically and $47 \%$ when treated surgically. ${ }^{3}$ The most dreaded complication is pump failure or cardiogenic shock, which is the single most important determinant of operative mortality. ${ }^{1,2}$ Patch closure of the VSD can effectively close the defect and eliminate the shunt. However, for patients with extensive infarction and pump failure, there is a need for an effective mechanical support strategy, and external ventricular assist devices have been successfully used, maintaining more physiologic hemodynamics with minimum doses of drugs. ${ }^{4}$ To our knowledge, use of an implantable LVAD for postinfarction VSD has not been previously reported.

Initially, patients with acute myocardial infarction (AMI) were not thought to be good candidates for implantable LVAD insertion because of the technical problems in securing the inflow cannula to the necrotic infarcted myocardium. ${ }^{4,5}$ However, with experience, ${ }^{5}$ we found that patients with AMI could be safely bridged to transplantation. On that basis, we elected to use the implantable LVAD as a bridge to transplantation for patients with post-AMI VSD instead of an external ventricular assist device. In particular, these patients might have high preformed antibodies from prior blood transfusions, and the implantable devices allow for hospital discharge should the patient require prolonged support. Both patients demonstrated the typical pattern we have observed in patients with AMI involving the left main or left anterior descending coronary artery. The right ventricle functioned well because it was not involved in the infarction.

Although we have speculated that the total artificial heart (TAH) might be the ideal strategy for patients with post-AMI $\mathrm{VSD},{ }^{3}$ on the basis of our experience, the implantable LVAD might be an equally effective strategy for bridge to transplantation 
or permanent implantation. Also, the AMI heart is not dilated and might make proper fitting of the TAH difficult. However, postAMI ventricular arrhythmias might complicate LVAD patient management, although this was not a problem for these 2 patients, and theoretically favors TAH use.

\section{References}

1. Crenshaw BS, Granger CB, Birnbaum Y, Pieper KS, Morris DC, Kleinman NS, et al. Risk factors, angiographic patterns, and outcomes in patient with ventricular septal defect complicating acute myocardial infarction. GUSTO-I (Global Utilization of Streptokinase and TPA for Occluded Coronary Arteries) Trial Investigators. Circulation. 2000; 101:27-32.
2. David T, Dale L, Sun Z. Postinfarction ventricular septal rupture: repair by endocardial patch with infarct exclusion. $J$ Thorac Cardiovasc Surg. 1995;110:1315-22.

3. McCarthy PM, Fukamachi K, Fukumura F, Muramoto K, Golding LAR, Harasaki H. The Cleveland Clinic-Nimbus total artificial heart: in vivo hemodynamic performance in calves and preclinical studies. J Thorac Cardiovasc Surg. 1994;108:420-8.

4. Nasu M, Shinkai M, Fuhuwara H, Sono I, Okada Y, Myiamoto S, et al. Recovery of end-stage organ dysfunction by circulatory assist. ASAIO Trans. 1991;37:M345-7.

5. McCarthy PM, Smedira NG, Vargo R, Goormastic M, Hobbs R, Starling R, et al. One hundred patients with the HeartMate left ventricular assist device: evolving concepts and technology. J Thorac Cardiovasc Surg. 1998;115:904-12. 